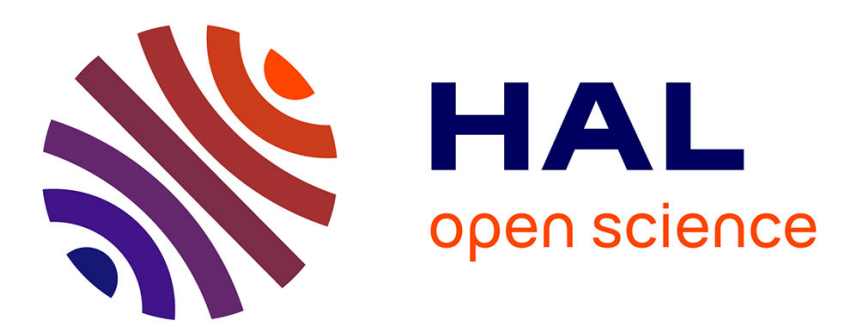

\title{
Velocity measurements based on shadowgraph-like image correlations in a cavitating micro-channel flow
}

Cyril Mauger, Loïc Méès, Marc Michard, Michel Lance

\section{To cite this version:}

Cyril Mauger, Loïc Méès, Marc Michard, Michel Lance. Velocity measurements based on shadowgraphlike image correlations in a cavitating micro-channel flow. International Journal of Multiphase Flow, 2014, 58, pp.301-312. 10.1016/j.ijmultiphaseflow.2013.10.004 . hal-01166554

\section{HAL Id: hal-01166554 https://hal.science/hal-01166554}

Submitted on 23 Jun 2015

HAL is a multi-disciplinary open access archive for the deposit and dissemination of scientific research documents, whether they are published or not. The documents may come from teaching and research institutions in France or abroad, or from public or private research centers.
L'archive ouverte pluridisciplinaire HAL, est destinée au dépôt et à la diffusion de documents scientifiques de niveau recherche, publiés ou non, émanant des établissements d'enseignement et de recherche français ou étrangers, des laboratoires publics ou privés. 


\title{
Velocity measurements based on shadowgraph-like image correlations in a cavitating micro-channel flow
}

\author{
C. Mauger, L. Méès, M. Michard, M. Lance \\ Laboratoire de Mécanique des Fluides et d'Acoustique (LMFA), CNRS UMR 5509 - \\ École Centrale de Lyon - INSA de Lyon - Université Claude Bernard - Lyon 1, Écully, \\ France \\ Corresponding author: Cyril Mauger, tel : 33 (0)4 72436164 , email: \\ cyril.mauger@insa-lyon.fr
}

\begin{abstract}
Cavitation is generally known for its drawbacks (noise, vibration, damage). However, it may play a beneficial role in the particular case of fuel injection, by enhancing atomization processes or reducing nozzle fouling. Studying cavitation in real injection configuration is therefore of great interest, yet tricky because of high pressure, high speed velocity, small dimensions and lack of optical access for instance. In this paper, the authors proposed a simplified and transparent 2D micro-channel (200-400 $\mu \mathrm{m})$, supplied with test oil at lower pressure $(6 \mathrm{MPa})$, allowing the use of non-intrusive and accurate optical measurement techniques. A shadowgraph-like imaging arrangement is presented. It makes it possible to visualize vapor formations as well as density gradients (refractive index gradients) in the liquid phase, including scrambled grey-level structures connected to turbulence. This optical technique has been already discussed in a previous paper (Mauger et al., 2012), together with a Schlieren and an interferometric imaging technique. In this paper, the grey-level structures connected with turbulence are considered more specifically to derive information on flow velocity. The grey-level structure displacement is visualized through couples of images recorded within a very short time delay (about $300 \mathrm{~ns}$ ). At first, space and space-time correlation functions are calculated to characterize the evolution of grey-level structures. Space-time correlations provide structure velocity that slightly under-estimates the real flow velocity deduced from flowmeter measurements. Since the grey-level structures remain correlated in time, a second velocity measurement method is applied. An image correlation
\end{abstract}


algorithm similar to those currently used in Particle Image Velocimetry (PIV) is used to extract velocity information, without seeding particles. In addition to the mean velocity of grey-level structures, this second method provides structure velocity fluctuations. In particular, an increase in structure velocity fluctuations is observed at the channel outlet for a critical normalized length of vapor cavities equals to 40-50\%, as expected for the real flow velocity fluctuations. The present study is completed by a parametric study on channel height and oil temperature. It is concluded that none of them significantly impact the critical normalized length for which the fluctuation increase is observed, even though the magnitude of these fluctuations is larger for the higher channel.

Keywords: Cavitation, shadowgraph, turbulence, channel flow, correlation, image correlation, Diesel injector

\section{Introduction}

Over the years, automotive industry standards have increasingly forced manufacturers to produce eco-friendly vehicles. During the last decades, heat engines have been improved, becoming less pollutant yet more efficient. The optimization of the thermodynamic cycle has played a key role in the improvement process. More precisely, increased knowledge of the internal aerodynamics of cylinders and enhanced injection systems have made it possible to better control fuel evaporation and mixture, and therefore fuel combustion. Nevertheless, in many respects, the atomization process at the injector outlet - in particular, the influence of the internal flow on atomization - is still not well understood.

The spray characteristics of Diesel injectors depend on the atomization processes in cylinders, and therefore, they depend on the velocity profile and turbulence inside the nozzle and at the nozzle exit (Birouk and Lekic, 2009). The spray characteristics are then likely influenced by the presence of cavitation in nozzle orifices. Using a backlit micro-PIV system in a real size transparent VCO nozzle, Chaves (2008) highlights that cavitation appears at the sharp edge inlet of the injection orifice. Vapor cavities extend along the orifice and bubbles seem to collapse in a very deterministic and localized manner. Velocity fields are also measured by using Particle Image Velocimetry (PIV). However, the introduction of seeding particles in the flow is potentially problematic: Particles can act as cavitation nuclei 
and, consequently, modify the conditions of cavitation inception. Different cavitation regimes are described in the literature, namely no cavitation, developing cavitation, super-cavitation and hydraulic flip (Sou et al., 2007). Most authors (Birouk and Lekic, 2009; Sou et al., 2007; Hiroyasu, 1991; Soteriou et al., 1995; Wu et al., 1995; Hiroyasu, 2000; Tamaki et al., 2001) show that the most favorable regime for spray atomization is super-cavitation while hydraulic flip is the worst in this respect (Sou et al., 2007; Hiroyasu, 1991; Bergwerk, 1959). It is considered that super-cavitation is reached when the vapor cavity length is 70 to $100 \%$ the orifice length (Sou et al., 2007). In super-cavitation condition, at least two mechanisms are supposed to influence spray formation. Firstly, cavitation may increase flow turbulence (Tamaki et al., 2001; He and Ruiz, 1995) through bubble collapse and pressure waves. Secondly, bubbles may reach the channel outlet and enhance directly the liquid jet atomization when they collapse (Sou et al., 2007). In order to provide useful information on the internal flow inside injection orifices, different experimental setups are presented in the literature. Flow investigation in a realistic geometry and real injection conditions is a difficult task because of high pressure, high velocities, small dimensions, lack of optical access and strong unsteadiness. As a result, most experimental investigations are carried out at lower pressure injection, in a simplified geometry and/or in up-scaled orifices. In a large-scale channel, He and Ruiz (1995) measure both cavitating and non-cavitating flows by means of a Laser Doppler Velocimeter (LDV). When cavitation occurs, they notice a difference in the mean streamwise velocity component profile near the inlet and a 10$20 \%$ increase in turbulence intensity behind vapor cavities. In order to investigate the relationship between vapor cavity length and turbulence, high speed visualizations and Particle Image Velocimetry (PIV) measurements are conducted by Stanley et al. (2008) in an up-scaled, sharp-edged, acrylic nozzle. In super-cavitation regime, vapor bubbles convected through the nozzle exit have a significant influence on the liquid jet structure and enhance the aerodynamic shear break-up of the jet. Turbulent Kinetic Energy (TKE) is shown to be strongly linked to vapor cavity length inside the nozzle. Sou et al. (2007) also use LDV in a 2D up-scaled channel. They suggest that the strong turbulence induced by the collapse of cavitation clouds near the exit plays a major role in ligament formation. They visualize cavitation in the nozzle and ligament formation at liquid jet interface simultaneously using a high-speed camera. They find that the formation of a ligament is often (but not systematically) preceded by the collapse of a cavitation cloud at the 
channel outlet. It seems that the size of a ligament is roughly proportional to the size of the vapor formation preceding it. However, Sou et al. (2008) report that the formation of ligaments induced by a collapse of bubbles is less observed in a cylindrical configuration than in a channel configuration. This can be explained by the greater difficulty in observing a flow in a cylindrical configuration. For that reason, 2D channel configurations are preferred by some authors to study cavitation formation. Winklhofer et al. (2001) investigate a cavitating flow in a micro-channel with backlit imaging. They measure velocity profiles with a fluorescence tracing method. Velocity profile measurements show that vapor formation in the channel inlet increases flow velocities near the liquid-vapor interface. Winklhofer et al. (2001) also reconstruct the pressure field inside the channel by means of a Mach-Zehnder interferometer arrangement. For different cavitation regimes, they compare the pressure field and hydraulic behavior. They notice that the flow is choked after super-cavitation. These studies suggest that the turbulence induced by cavitation plays a major role in spray formation. It is clear that supercavitation is the most favorable regime to enhance atomization. Further investigations are required to highlight cavitation/atomization dependency.

Although observing cavitation inside nozzle orifices is a difficult task, experimental data, especially at the nozzle outlet, are needed to enhance our general knowledge on high pressure injection processes and to provide reliable initial conditions for numerical simulations (Ménard et al., 2007; Gorokhovski and Herrmann, 2008; Marcer et al., 2008; Lebas et al., 2009). Obtaining quantitative information as velocity profiles is even more complicated. Consequently, a simplified experimental configuration is considered in this paper. A 2D micro-channel permanent flow is visualized by using a shadowgraph-like imaging setup, based on a backlit illumination and sensitive to refractive index gradients. This setup has already been used together with alternative imaging techniques to study cavitation inception, under conditions close to those of direct injection in a $400 \mu \mathrm{m}$ high microchannel (Mauger et al., 2012). In this paper, couples of shadowgraph-like images are considered to perform velocity measurements in a $2 \mathrm{D}$ microchannel, without seeding particles. The experimental setup is presented in Section 2. Shadowgraph-like images of the channel flow are presented in Section 3 together with the result of space and space-time correlations. Section 4 is dedicated to the measurement of mean velocities and fluctuations, based on an image correlation algorithm for a channel height between 200 $\mu \mathrm{m}$ and $400 \mu \mathrm{m}$. 


\section{Experimental setup}

In the present study, cavitating flow is investigated in a quasi-2D orifice (micro-channel). The micro-channel consists of two separated metal sheets, sandwiched between a pair of glass windows (Figure 1). It is continuously supplied with fuel (test oil SHELL V-Oel-1404) through holes directly drilled into the glass windows. Oil pressure levels are measured $40 \mathrm{~mm}$ upstream and downstream of the channel using metal thin film sensors. A variable area meter measures the flow rate. Oil temperature is regulated by an air/oil heat exchanger and controlled by a T-type thermocouple. In the following, the flow temperature upstream the channel is $T=24^{\circ} \mathrm{C}$.

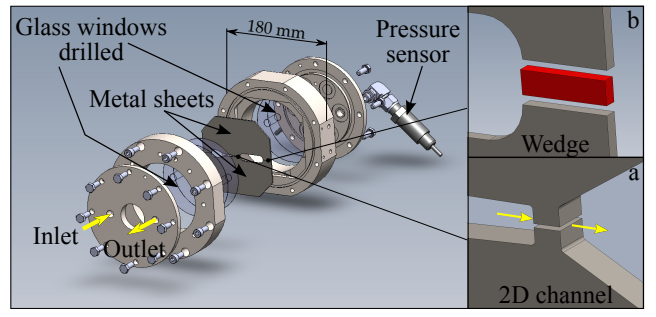

Figure 1: Exploded view of the 2D micro-channel.

The use of two separated metal sheets makes it possible to better control channel geometry and wall roughness. A Scanning Electron Microscope (SEM) is used to measure channel dimensions. The channel is about $388 \mu \mathrm{m}$ high and 1,475 $\mathrm{mm}$ long, with an area reduction of $5 \%$ between the inlet and the outlet. The channel depth is $L=2 \mathrm{~mm}$. Channel inlets are very sharp $(r \simeq 10 \mu \mathrm{m})$. The surfaces constituting the channel walls have been mirrorpolished. Their roughness is characterized by using an optical profilometer, by a mean arithmetic roughness parameter $R_{a}<0.1 \mu \mathrm{m}$.

Different flow conditions are obtained maintaining upstream pressure $p_{\text {up }}$ constant and varying downstream pressure $p_{\text {down }}$. Depending on the imposed pressure drop $\Delta p=p_{\text {up }}-p_{\text {down }}$, three flow regimes are identified, namely single-phase flow, cavitating flow and choked flow. Cavitating flow is divided in three steps: Cavitation inception, cavitation development and super-cavitation. Since the discharge volume is liquid, no hydraulic flip can be observed.

The channel flow is visualized using a shadowgraph-like imaging technique which is based on a backlit illumination, allowing the detection of vapor 
bubbles and cavities, and providing qualitative information on density (refractive index) gradients.

The small size of the channel requires the use of large optical magnification. With flow velocities up to $80 \mathrm{~m} \cdot \mathrm{s}^{-1}$, an extremely short light pulse is needed to optically freeze the flow. In addition, an incoherent light source is required to avoid speckle on images. Figure 2 presents the optical arrangement.

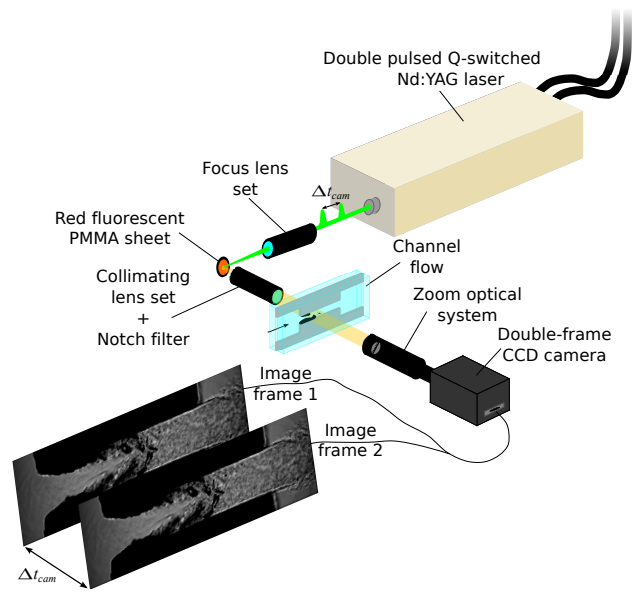

Figure 2: Shadowgraph-like optical arrangement.

An adequate light source is generated by focusing the second harmonic of a Nd:YAG pulsed laser (wavelength $\lambda_{Y A G}=532 \mathrm{~nm}$ and pulse duration $=6 \mathrm{~ns}$ ) on a fluorescent polymethyl-methacrylate (PMMA) sheet. The fluorescent emission is collimated and the remaining laser light is filtered using a band reject filter (Notch filter $\lambda_{\text {Notch }}=533 \pm 8 \mathrm{~nm}$ ). The Nd:YAG dual head laser helps to produce time delayed light pulses. Each incoherent light pulse lasts $12 \mathrm{~ns}$ (FWHM) and has a broad spectrum (Figure 3). The time delay between two pulses can be adjusted, down to $\Delta t_{\text {min }} \simeq 30 \mathrm{~ns}$. Images are recorded by means of an optical zoom system (OPTEM 125C) on a $2048 \times 2048$ pixels, 10-bit CCD camera. The resulting magnification is about 6.4 with a resolution of $1.15 \mu \mathrm{m} \cdot \mathrm{px}^{-1}$. Using the double-frame mode of the camera and the dual pulse system of the laser, couples of images separated by $\Delta t_{\text {cam min }} \simeq 200 \mathrm{~ns}$ or more are recorded. This optical arrangement permits the tracking of rapid events, like bubble dynamics, and the production of velocity information. 

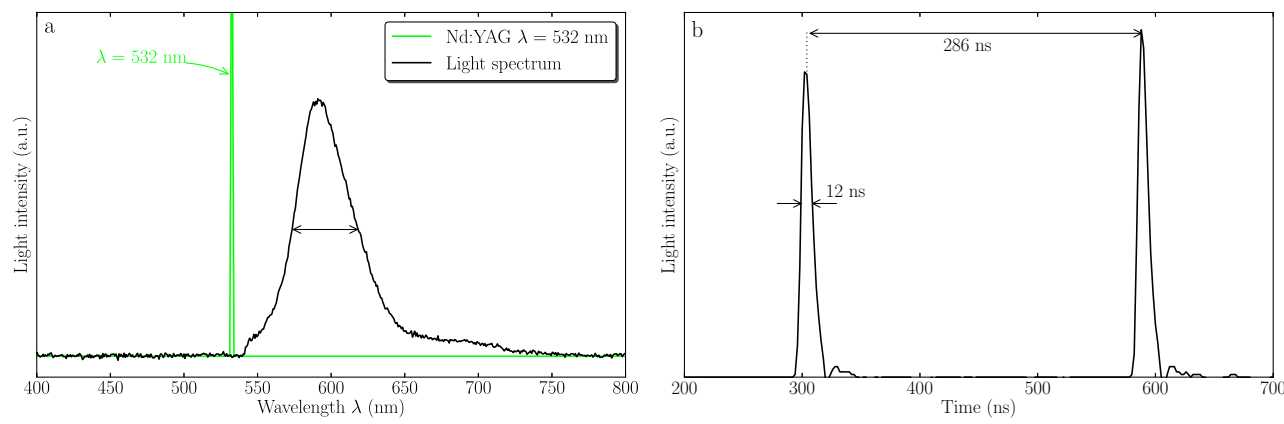

Figure 3: a: Spectrum measured by means of an optical fiber-based mini-spectrometer. b: Light source temporal profile measured by means of a fast photo-diode.

\section{Shadowgraph-like images and correlations}

\subsection{Images description}

Figure 4 displays examples of instantaneous images. Flow direction is left to right. Each image number is reported on a graph (Figure 14) which represents the mass flow rate $\dot{m}$ versus the square root of the pressure drop $\sqrt{\Delta p}$.

On images, bright areas correspond to liquid phases whereas dark areas correspond to vapor formations or walls, as expected on backlit images. Greylevel variations are also observed, due to a shadowgraph effect revealing density (refractive index) gradients. Density gradients are associated to pressure or/and temperature variations. The optical arrangement can therefore be viewed as a backlit imaging arrangement which is sensitive to density gradients but differs somewhat from shadowgraph techniques, strickly speaking, hence the name of "shadowgraph-like imaging". Figure 4 reveals the following features that are detailed and discussed by Mauger et al. (2012):

a- streamlines (density gradients) that originate from an unexpected thermal marking of the flow arising far upstream from the channel and only visible in low velocity (low pressure difference) conditions;

b- shear layers at the corners due to a density difference between the separation zones and the main flow;

c- out-going jet boundaries, where both temperature and pressure gradients are involved. The temperature gradient would be due to the viscous heating of the fluid near the walls. The pressure gradient 
would be associated to the pressure difference between the out-going jet and the downstream chamber or to local pressure drops inside vortices generated in the jet shear layers;

d- grey-level random-like variations in the wake of the separation zones. They appear as structures developing from the walls to the center of the channel where they join together. These grey-level structures are connected to the turbulence developing in the flow;

e- cavitation inception in the shear layers under the combined effect of the depression induced by flow detachment at the channel inlet and vortices caused by instabilities in the shear layers. Mauger et al. (2012) combine several optical methods to support this scenario which has also been observed in a scale-up configuration (Iben et al., 2011);

f- pressure waves caused by vapor bubble collapse;

g- vapor bubble detachments.

Shadowgraph effects provide an additional amount of information but they also make image interpretation more difficult, as discussed in Mauger et al. (2012) study. The grey-level random-like variations mentioned above are present in most images. These intensity variations are associated to density (refractive index) variations caused by turbulent structures inside the flow. Although the shadowgraph effects provide information on refractive index variations which is integrated along the whole channel depth and essentially qualitative, the grey-level structures are linked to the turbulent structures convected by the flow. Their displacements are clearly visible from a quick glance at two successive images. In the following, space and spacetime correlation functions are used to study these displacements for different flow conditions.

\subsection{Space and space-time correlations}

First, image grey-level variations are obtained by subtracting the mean image of a 50-image series from each image. In the present case, the flow is supposed to be two-dimensional. $\psi^{\prime}(\mathbf{x}, t)$ is the grey-level variation at

position $\mathbf{x}$ and time $t$. The space-time correlation function at position $\mathbf{x}$ and $\mathbf{x}+\xi$ is defined by: 

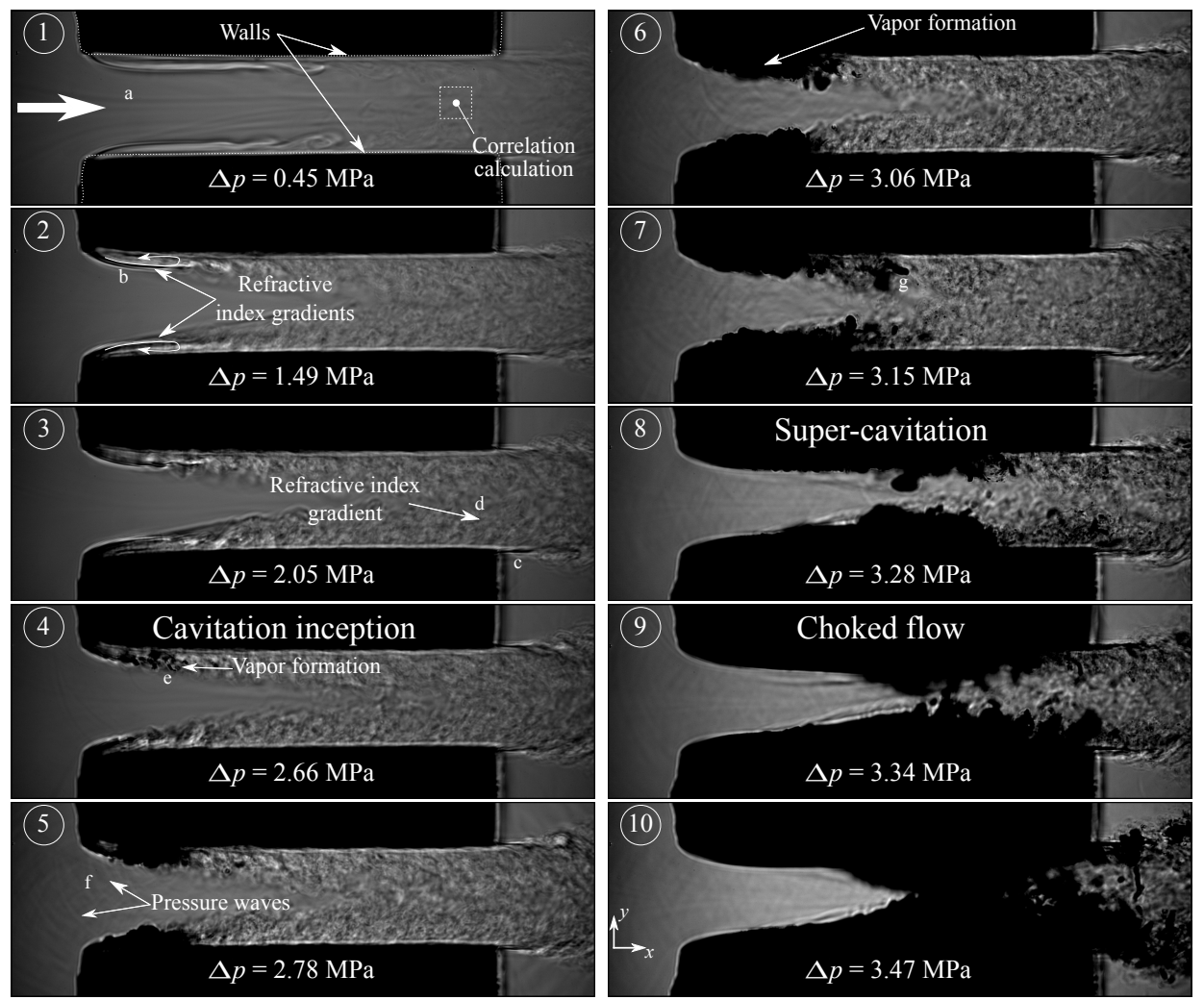

Figure 4: Examples of instantaneous shadowgraph-like images. The channel height is $388 \mu \mathrm{m} . p_{u p}=5.00 \mathrm{MPa}, T=24^{\circ} \mathrm{C}$. 


$$
R(\xi, \mathbf{x}, \Delta t)=\frac{\left\langle\psi^{\prime}(\mathbf{x}, t) \psi^{\prime}(\mathbf{x}+\xi, t)\right\rangle}{\sqrt{\left\langle\psi^{\prime}(\mathbf{x}, t)^{2}\right\rangle} \sqrt{\left\langle\psi^{\prime}(\mathbf{x}+\xi, t)^{2}\right\rangle}}
$$

where $\langle$.$\rangle is the time-averaging operator and \Delta t$ the time delay between two images.

\subsubsection{Space correlations}

The space correlation $R(\xi, \mathbf{x}, \Delta t=0)$ is first considered. Figure 5a displays the iso-contours of the space correlation function calculated in the flow region shown in image number 1 of Figure 4 , but for $\Delta p=2.71 \mathrm{Mpa}$. A correlation peak is clearly visible in the center of the map. An integral length scale based on density fluctuations corresponding to $R(\xi, \mathbf{x}, \Delta t=0)$ can be defined as:

$$
L_{n}(\mathbf{x})=\int_{0}^{\infty} R(\xi, \mathbf{x}, \Delta t=0) d \xi
$$

An integral length scale can be deduced for the streamwise direction $\left(L_{n x}\right)$ or the cross-streamwise direction $\left(L_{n y}\right)$. In practice, the calculation of the integral length scale cannot extend to infinity. It is then interrupted when a zero value is achieved. Convergence is hardly obtained with only 50 images. The noise that can be seen in Figure 5a accounts for the apparent lack of statistics. At channel outlet, the turbulence is assumed to be locally homogeneous. In order to increase the statistical convergence of $R(\xi, \mathbf{x}, \Delta t)$, the correlation function is calculated for pixels located close to the initial point, i.e. for pixels in a $11 \times 11$ pixel square, centered on the initial pixel. An example of space correlation function averaged over 121 points is shown in Figure 5b. The positive iso-values of the space correlation function are almost circular, showing a quasi-isotropic pattern. This observation is in line with the results obtained by Kim and Hussain (1992) for space-correlations based on pressure fluctuations in a fully established channel flow.

In Figure 6, the integral length scales $L_{n x}$ and $L_{n y}$ are plotted against the Reynolds number $R e$, from low pressure drop condition (lower $R e$ ) to super-cavitation (higher $R e$ ). The Reynolds number is defined as:

$$
R e=\frac{\rho U_{M} D_{h}}{\mu}
$$




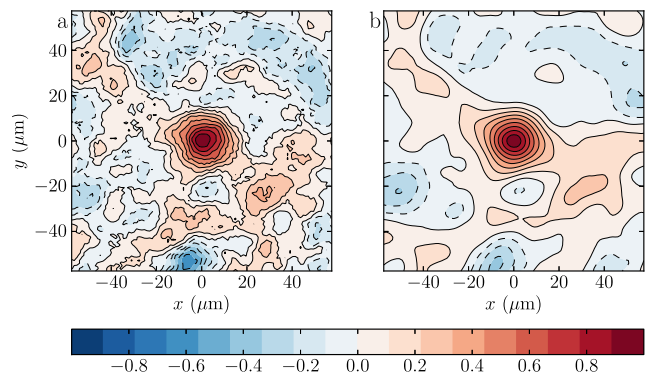

Figure 5: Iso-contours of the space correlation function $R(\xi, \mathbf{x}, \Delta t=0)$ at the channel outlet centerline $(0.9 L) \cdot p_{u p}=5.00 \mathrm{MPa}, \Delta p=2.71 \mathrm{MPa}, T=24^{\circ} \mathrm{C}$. a: Single point correlation. b: Correlation function averaged over 121 points.

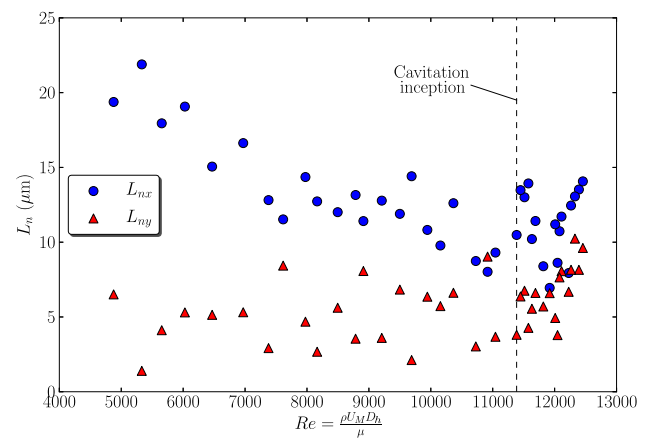

Figure 6: Evolution of the integral length scales $L_{n x}$ and $L_{n y}$ versus the Reynolds number $R e$ at the channel outlet centerline $(0.9 L) . p_{u p}=5.00 \mathrm{MPa}, T=24^{\circ} \mathrm{C}$.

where $\rho$ and $\mu$ are the density and the dynamic viscosity of the test oil, respectively. The values of these parameters are given by Ndiaye et al. (2012) as a function of temperature and pressure. $D_{h}$ is the hydraulic diameter of the channel and $U_{M}$ the mean velocity inside the channel.

The same averaging method as the one described above is used to increase the level of statistical convergence for $L_{n x}$ and $L_{n y}$. In Figure 6, the integral length scale $L_{n x}$ decreases with increasing Reynolds number until cavitation inception. In the cross-streamwise direction, $L_{n y}$ remains almost constant. Once cavitation appears, $L_{n x}$ and $L_{n y}$ seem to slightly increase. For greater values of the Reynolds number, in the super cavitation regime, the presence of cavitation in the region of interest (ROI) does not allow the correlation calculation.

To go further in the analysis, grey-level structures are now investigated 


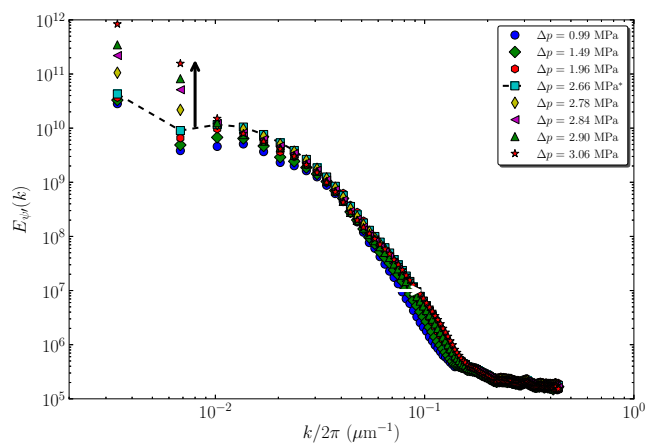

Figure 7: Grey-level variation spectrum for different flow conditions. ${ }^{*}$ Cavitation inception. $p_{u p}=5.00 \mathrm{MPa}, T=24^{\circ} \mathrm{C}$.

by using a two-dimensional discrete Fourier transform. The two-dimensional energy spectrum $\Delta(\mathbf{k})$ of the grey-level variation is defined as:

$$
\Delta(\mathbf{k})=\left(\frac{2 \pi}{a}\right)^{2}\left|\Psi^{\prime}(\mathbf{k})\right|^{2}
$$

where $\Psi^{\prime}(\mathbf{k})$ is the Fourier transform of the grey-level variation $\psi^{\prime}(\mathbf{x})$ and $a$ the ROI. Grey-level variations are assumed to be statistically homogeneous and isotropic, $\Delta(\mathbf{k})=\Delta(k)$. It is then useful to define another spectral function $E_{\psi^{\prime}}(k)$, which is the radial average of the two-dimensional spectrum $\Delta(\mathbf{k})$, multiplied by a factor $2 \pi k$ :

$$
E_{\psi^{\prime}}(k)=2 \pi k \Delta(k)=\int_{0}^{2 \pi} \Delta(k, \phi) k d \phi
$$

The ROI is a square of $256 \times 256$ pixels $\left(\simeq 295 \mu^{2}\right)$ located at the channel outlet. To reduce aliasing due to ROI boundaries, a Hann window function is applied. Examples of spectra are given in Figure 7 for different flow conditions. From single-phase flow to cavitation inception (from $\Delta p=0.99 \mathrm{MPa}$ to $\Delta p=2.66 \mathrm{Mpa}$ ), the spectra slightly move toward smaller structures (white arrow) and larger structures increase. After cavitation inception $(\Delta p \geq 2.78 \mathrm{MPa})$, spectrum distributions superimpose at small scales when the largest scales increase faster than before cavitation inception (black arrow). The occurrence of cavitation seems to have an influence on the evolution of integral length scales and on grey-level variation spectra. However, these behaviors have no obvious interpretation (not even for low $\Delta p$ before cavitation inception) and it is not clear yet if the behavior of the 

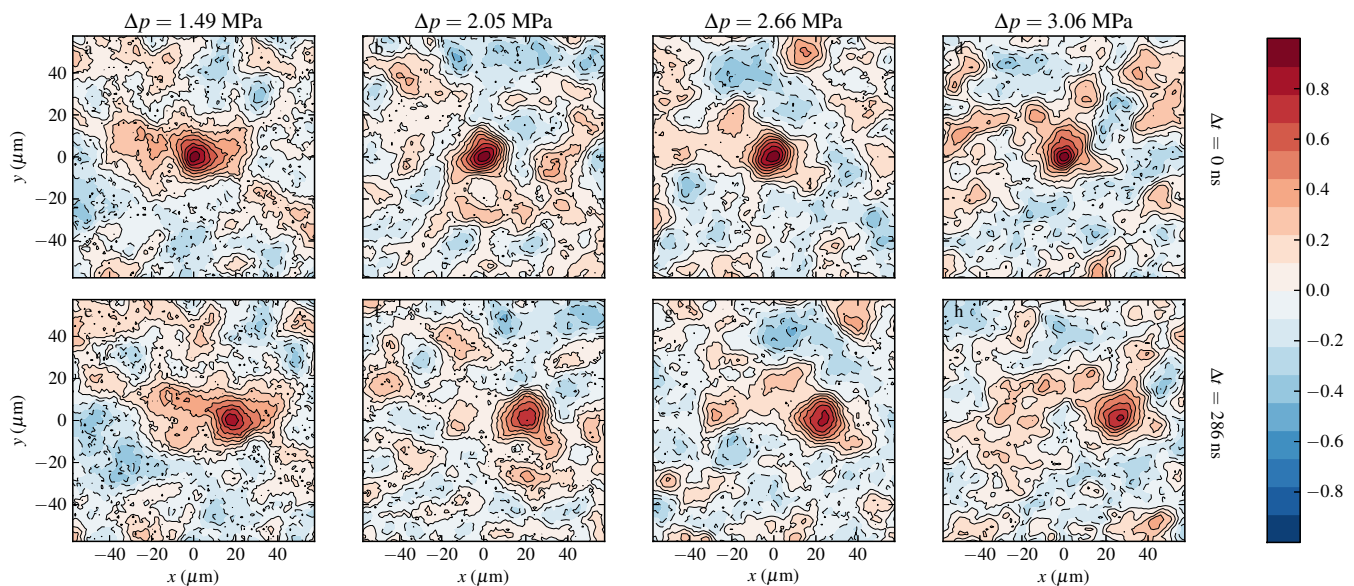

Figure 8: Iso-contours of the space-time correlation functions $R(\xi, \mathbf{x}, \Delta t=0 \mathrm{~ns})$ and $R(\xi, \mathbf{x}, \Delta t=286 \mathrm{~ns})$ at the channel exit centerline $0.9 L . p_{u p}=5.00 \mathrm{MPa}, T=24^{\circ} \mathrm{C}$. a and e: $\Delta p=1.49 \mathrm{MPa}, \mathrm{b}$ and f: $\Delta p=2.05 \mathrm{MPa}$, c and g: $\Delta p=2.66 \mathrm{MPa}, \mathrm{d}$ and h: $\Delta p=3.06 \mathrm{MPa}$.

grey-level structures can be directly linked to turbulent flow characteristics, such as pressure or density spectra. In the following, the displacement of the grey-level structures will be investigated through space-time correlation computations.

\subsubsection{Space-time correlations}

A quick glance at two successive images of the flow, recorded within a short time $\Delta t$ (less than $300 \mathrm{~ns}$ ), shows a clear displacement of the grey-level structures. Space-time correlation functions are now considered to determine whether this displacement can be used to obtain velocity information of the flow. In this paper, only one value of $\Delta t$ is considered $(\Delta t=286 \mathrm{~ns}$ ), which does not allow one to study the flow in terms of turbulence time scale. Nevertheless, the space-time correlation function for $\Delta t=286$ ns can be used to estimate an advection velocity of the turbulent structures. Examples of space-time correlations $R(\xi, \mathbf{x}, \Delta t=0 \mathrm{~ns})$ and $R(\xi, \mathbf{x}, \Delta t=286 \mathrm{~ns})$ are given in Figure 8 for different flow conditions inside the channel at streamwise location $x / L=0.9 L$.

Figure 8 displays examples of correlation functions for $\Delta t=0 \mathrm{~ns}$ (Figure 8a-d) and $\Delta t=286$ ns (Figure 8e-h), computed from a sample of images (or image couples) recorded in four different flow conditions $(\Delta p)$. In each case, the correlation function for $\Delta t=286 \mathrm{~ns}$ is similar to the one 
for $\Delta t=0 \mathrm{~ns}$, but correlation peaks are clearly shifted, mainly in the mean flow direction. The peaks are also slightly attenuated but their displacement can be easily quantified by a separation vector $\xi_{c}$ from which a grey-level structure advection velocity $U_{c}=\xi_{c} / \Delta t$ can be defined. No spatial averaging similar to the one applied in Figure $5 \mathrm{~b}$ is required to extract this advection velocity. In Figure 8, the structure displacement increases with the pressure drop. Figure 9 shows the structure advection velocity deduced from a larger sample of images. The streamwise and cross-streamwise components of the grey-level structure advection velocity $U_{c x}$ and $U_{c y}$ are plotted against the Reynolds number based on the mean velocity $U_{M}$ deduced from flow rate measurements. The cross-streamwise velocity component remains almost constant and close to zero. The streamwise velocity component increases linearly with the Reynolds number, as expected. $U_{c x}$ is close to the mean velocity $U_{M}$ deduced from flow rate measurements and represented by the black line in Figure 9. $U_{c x}$ is however lower than $U_{M}$ by about $10 \%$, in the whole range of measurement. Several explanations can be proposed to account for this discrepancy. It is assumed that the grey-level structures are connected to turbulence through density variations and a shadowgraph effect. Firstly, the advection velocity of the turbulent structures could be lower than the flow velocity. Secondly, the grey-level structures could move at a lower velocity than the turbulent structures. The shadowgraph effect being integrated along the whole depth of the channel (along light ray paths, perpendicular to images), it could be more sensitive to density variations, in particular flow areas for example - in the boundary layers near the glass windows, where the velocity is lower. The discrepancy could also be attributed to the flow rate measurement which is performed upstream the channel. The sealing of the channel is achieved by a direct glass/metal contact to avoid unwanted cavitation formations, due to the use of glue for example. The sealing may not be perfect and a part of the flow could be diverted outside the channel, between the glass windows and the metal sheets. Finally, the mean grey-level structure velocities presented in Figure 9 have been obtained from a flow area located in the channel centerline, as shown in Figure 4-1. Later in the paper, in Section 4.2, a possible bias in structure velocity measurement in the centerline of the channel will be discussed. From the results presented in this paper, the equivalence between the grey-level structure velocity and the real flow velocity cannot be rigorously evidenced. However, the behavior of the mean velocity measured from the grey-level structures is consistent with what is expected for the real flow velocity, 


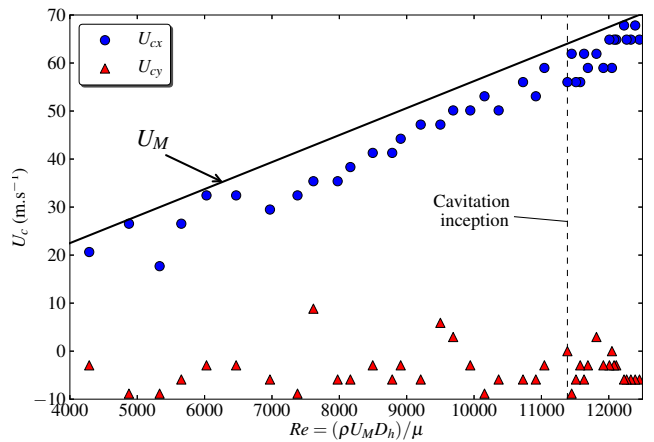

Figure 9: Velocities deduced from correlation peak displacement $U_{c x}$ and $U_{c y}$ versus the Reynolds number Re at the channel exit centerline $0.9 L$. $p_{u p}=5.00 \mathrm{MPa}, T=24^{\circ} \mathrm{C}$. Black line corresponds to the mean velocity $U_{M}$, deduced from flow rate measurements.

justifying further analysis of the grey-level structure velocity in the following.

The main result presented in this section is that the space-time correlation function calculations show that the grey-level structures are still very well correlated after a time delay $\Delta t=286$ ns. Then, an image correlation algorithm can be applied to grey-level variations in order to extract velocity information, without seeding particles, i.e. without additional cavitation nuclei. In addition to the mean velocity already obtained from space-time correlation function, the image correlation algorithm provides information on velocity fluctuations which are of interest considering their potential impact on atomization processes.

\section{Shadowgraph-like image correlations}

In the following, the term "structure velocity" refers to the velocity of grey-level structures and the term "flow velocity" designates the real flow velocity, whether it is unknown or deduced from flowmeter measurements. The equality between these velocities is not rigorously evidenced but based on the presented results, structure velocity is attempted to be representative of flow velocity.

\subsection{Image correlation algorithm}

For each couple of images, the turbulent structure displacement can be clearly seen from an image to the other. The displacement field is calculated using a custom-written particle image velocimetry program implemented as 
an ImageJ plugin (http://rsb.info.nih.gov/ij) by Tseng et al. (2012). The package of PIV software is available at https://sites.google.com/ site/qingzongtseng/piv. The displacement field is performed through an iterative scheme. The spacing between each interrogation window for the last pass is $12 \mathrm{px}$, resulting in a final grid size for the displacement field of $14 \mu \mathrm{m} \times 14 \mu \mathrm{m}$. The image processing makes it possible to determine structure displacement in the whole channel with the exception of zones where walls or vapor formations are present.

A data post-processing is required to eliminate erroneous displacement vectors due to zones that are free from structures. The post-processing is applied when the standard deviation of grey-levels in an interrogation window $(12 \mathrm{px} \times 12 \mathrm{px})$ is below a critical value - typically 5 . It may happen that this threshold method removes vectors that should be preserved. If so, a new vector is calculated from the neighbor vectors when they are not equal to zero. The quantity of vectors recalculated is about $3 \%$ of the total number of vectors. Channel dimensions are well known thanks to SEM visualizations and the time delay between two light pulses has been accurately measured with a fast photodiode. The velocity field of the turbulent structures can therefore be reconstructed. Figure 10 presents a schematic of image processing and post-processing.

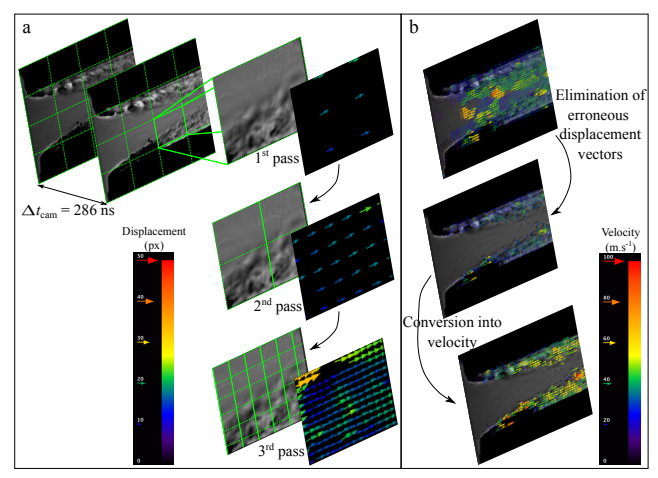

Figure 10: Schematic of image processing (a) (Tseng et al., 2012) and post-processing (b).

The image processing is applied to series of 50 images recorded in the same conditions. For each series, the mean structure velocity $\left\langle U_{\psi^{\prime}}\right\rangle$ and root mean square (RMS) of structure velocity are calculated. No structure velocity can be obtained in zones that are free from structures and in those with vapor formations. For a given zone, the images from which no velocity is obtained 
are not taken into account in the mean and RMS calculations. Also, mean velocity and RMS are ignored when they are based on a sample smaller than 10 (velocity information deduced from less than $20 \%$ of all images). An example of partial structure velocity field combined with probability of cavitation occurrence is shown in Figure 11.

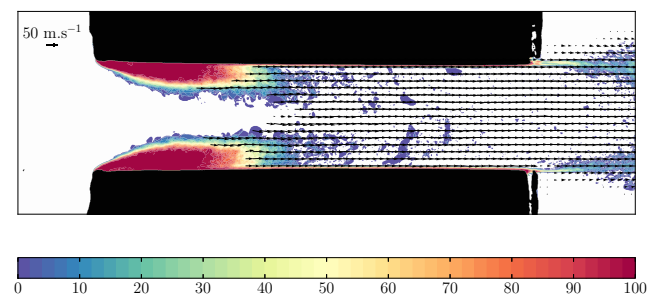

Figure 11: Probability of cavitation occurrence combined with partial structure velocity field. $p_{\text {up }}=5.00 \mathrm{MPa}, \Delta p=3.06 \mathrm{MPa}, T=24^{\circ} \mathrm{C}$.

Since vapor cavities develop in the channel as the pressure difference increases, velocity information is not taken into account either when the probability of cavitation occurrence is higher than $50 \%$ in the ROI.

\subsection{Structure velocity profiles}

Figure 12 shows streamwise structure velocity profiles $\left\langle U_{\psi^{\prime} x}\right\rangle$ averaged over a series of images at different locations inside the channel $(0.4 \mathrm{~L}, 0.65 \mathrm{~L}$ and $0.9 L)$. The profiles at $0.4 L$ are incomplete because structures are not present through the whole height of the channel at this location. Closer to the channel inlet, for $x<0.4 L$, no structure velocity measurement is performed because of limited statistics (few structures).

At $0.4 L$ (Figure 12b), a global increase in structure velocity with increasing $\Delta p$ is observed, as expected. The velocity information is only partial at this location. Near the centerline, the profiles are cut-off in the absence of structures. For $\Delta p=3.06 \mathrm{MPa}$ and $\Delta p=3.28 \mathrm{MPa}$, the edges of profiles are also cut-off due to the presence of vapor cavities near the walls.

At $0.65 \mathrm{~L}$ and $0.9 \mathrm{~L}$ (Figure $12 \mathrm{c}-\mathrm{d}$ ), the profiles are almost flat. The profiles for $\Delta p=1.49 \mathrm{MPa}$ are also incomplete at these locations, due to the lack of structures. A structure velocity deficit is noticed at the center of the profile for $\Delta p=3.06 \mathrm{MPa}$. At these locations, the two boundary layers merge, possibly leading to a deformation of the structures within the time delay between the two images, which may bias the structure velocity measurements. 
At $0.9 L$, the profiles for $\Delta p=3.06 \mathrm{MPa}$ and $\Delta p=3.28 \mathrm{MPa}$ are quite similar, testifying of the imminence of the choked flow.

Figure 13 shows cross-streamwise velocity profiles $\left\langle U_{\psi^{\prime} y}\right\rangle$ averaged over a series of images at different locations inside the channel $(0.1 h, 0.3 h$ and $0.5 h)$.

These structure velocity profiles appear to be very noisy compared to the streamwise velocity profiles because the corresponding displacements (in $y$ direction) are in the order of one or two pixels only. At $0.1 \mathrm{~h}$ (Figure 13b) cross-streamwise structure velocity profiles $\left\langle U_{\psi^{\prime} y}\right\rangle$ are negative on average. This means that the structures slightly move toward the channel walls. At the channel inlet, recirculation zones (in absence of cavitation) or cavitation formations are followed by a flow reattachment that may force the turbulent structures to move toward the walls. The situation differs here from the usual displacement of turbulent structures, from walls to the center, when turbulence generated near a wall without flow separation is considered. For $\Delta p=3.06 \mathrm{MPa}$ and $\Delta p=3.28 \mathrm{MPa}$, after cavitation inception, the profiles are cut-off near the channel inlet, due to the presence of vapor cavities. At $y=0.3 h$ and $y=0.5 h$, in Figure $13 \mathrm{c}$ and $13 \mathrm{~d}$ respectively, the same cut-off is noticed for the same reason. For $\Delta p=1.49 \mathrm{MPa}$ and $\Delta p=2.66 \mathrm{MPa}$, the profiles are also cut-off in the absence of grey-level structures.

At $0.3 h$ (Figure 13b), cross-streamwise structure velocities $\left\langle U_{\psi^{\prime} y}\right\rangle$ are still negative in the wake of the shear layer (or vapor cavities), meaning that the grey-level structures move toward the channel walls in this region (reattached flow). The structure velocity then increases regularly throughout the channel to reach zero at the outlet. At $0.5 h$ (Figure 13c), the cross-streamwise structure velocity $\left\langle U_{\psi^{\prime} y}\right\rangle$ is about zero in average, as expected for flow velocity in the centerline of the channel.

The velocity profiles show that the grey-level structures essentially move in the flow direction $x$, as it should be for flow velocity. The cross streamwise structure velocity $\left\langle U_{\psi^{\prime} y}\right\rangle$ is close to zero everywhere. In (Figure 14), a mass flow rate is deduced by integrating the structures velocity profiles near the channel outlet and compared to the mass flow rate measured with the flowmeter. The former underestimates the latter by about $6 \%$. Let us note that the structure velocity seems to be closer to the real flow velocity than in Figure 9 where a $10 \%$ discrepancy was found. In Figure 9, the structure velocities were obtained at the channel centerline, where a deficit in structure velocity is observed (Figure 12). Reversely, in Figure 14, the whole profiles 
are integrated to derive a flow rate, minimizing the effect of this deficit on the results. The difference between flow rates deduced from structure velocity profiles and measured by flow meter is bigger for the largest pressure drop considered in the Figure 14 , when the flow is choked $\left(\sqrt{\Delta} p \geq 1.82 \mathrm{MPa}^{0.5}\right)$. In choked flow condition, the occurrence of vapor cavities at the channel outlet increases, reducing the statistical reliability of the results. In addition, pressure waves associated to bubble collapses take part in the grey-level structures and probably distort the image correlation result or the grey-level structures themselves.

The experimental setup presented here does not make it possible to study the influence of the internal flow on spray formation because the discharge volume is liquid. Nevertheless, structure velocity fluctuations at the channel outlet can be investigated depending on flow conditions.

\subsection{Structure velocity fluctuations}

In Section 4.2, velocity information on advected grey-level structures has been obtained from shadowgraph-like images by using an image correlation algorithm. At the channel outlet, the measured structure velocities are consistent with the flowmeter measurements (Figure 14). It is then assumed that structure velocities are representative of real flow velocities and results are further analyzed in this section to extract structure velocity fluctuations. These fluctuations are derived from the flow area delimited by white dashes in Figure 15. The root mean squares of structure velocity $\sigma_{x}$ and $\sigma_{y}$ as well as the mean streamwise structure velocity $\left\langle U_{\psi^{\prime} x}\right\rangle$, are first averaged in space over this area leading to $\overline{\sigma_{x}}, \overline{\sigma_{y}}$ and $\overline{\left\langle U_{\psi^{\prime} x}\right\rangle}$ respectively. Relative fluctuations are then considered through the ratios $\varsigma_{x}$ and $\varsigma_{y}$ between the averaged root mean squares of structure velocity and the averaged streamwise mean structure velocity, that is

$$
\varsigma_{x}=\overline{\sigma_{x}} / \overline{\left\langle U_{\psi^{\prime} x}\right\rangle} \quad \text { and } \quad \varsigma_{y}=\overline{\sigma_{y}} / \overline{\left\langle U_{\psi^{\prime} x}\right\rangle}
$$

The streamwise and cross-streamwise structure velocity fluctuations are plotted against the Reynolds number in Figure 16a. The Reynolds number is not based on the structure velocity $\left\langle U_{\psi^{\prime} x}\right\rangle$ resulting from image correlation because this velocity measurement is not reliable in choked flow condition, as mentioned in the previous section. The Reynolds number is based on the mean velocity $U_{M}$ deduced from the flow rate measurements. In Figure 16a, the streamwise and cross-streamwise fluctuations evolve similarly but $\varsigma_{y}$ is 
lower than $\varsigma_{x}$. From $R e=5,000$ to 12,000 , the relative root mean square of structure velocity increases slowly for both streamwise and cross-streamwise directions. $\varsigma_{x}$ and $\varsigma_{y}$ raise from 7 to $10 \%$ and from 5 to $7 \%$, respectively. For $R e>12,000$ and until the choked flow regime, $\varsigma_{x}$ leaps by more than $34 \%$ and $\varsigma_{y}$ by more than $38 \%$. The sudden rise of structure velocity fluctuations does not occur at cavitation inception but for $R e=12,000$ when cavitation is already well developed in the channel.

It is common practice to introduce an dimensionless cavitation number when studying a cavitating flow. In Figure $16 \mathrm{~b}, \varsigma_{x}$ and $\varsigma_{y}$ are plotted against the cavitation number $K_{N}$ defined by Nurick (1976) as:

$$
K_{N}=\frac{p_{u p}-p_{\text {sat }}}{\Delta p}
$$

where $p_{\text {sat }}$ is the saturated vapor pressure of the test oil which is approximately equal to $10 \mathrm{~Pa}$ (Chora̧żewski et al., 2012). $p_{\text {sat }}$ can therefore be disregarded. Figure 16b (right to left for decreasing $K_{N}$ ) does not help us to better distinguish fluctuations in regards of developing cavitation inside the channel. Nevertheless, the sudden rise seems to appear for $K_{N}=1,7$.

A practical way of analyzing cavitation influence on velocity fluctuations is to plot $\varsigma_{i}$ versus the normalized length of vapor cavities $l_{\text {norm }}\left(l_{\text {cav }} / L\right)$. $l_{\text {cav }}$ is obtained from a series of images at each flow condition. On shadowgraph-like images, vapor appears in dark and liquid in grey-level variations depending on refractive index gradients. To eliminate refractive index gradients, segmented images are obtained by applying a threshold. Structure velocity fluctuations are plotted against the normalized cavity length $l_{\text {norm }}$ in Figure 16c, from cavitation inception to choked flow condition. Structure velocity fluctuations increase slowly from cavitation inception (lowest values of $l_{\text {norm }}$ ) to $l_{\text {norm }}$ equal to about $40-50 \%$. A more significant increase of fluctuations is observed for $l_{\text {norm }} \geq 50 \%$. Thus, velocity fluctuations at the channel outlet seem to be strongly affected by cavitation as soon as the vapor cavities reach the middle of the channel. This suggests that, in a real injector configuration, vapor formation may start to improve fuel atomization by increasing velocity fluctuations at this stage of cavitation development. Nevertheless, this hypothesis cannot be confirmed by the present experimental setup since it does not produce any spray (liquid volume discharge). Super-cavitation regime is known as the regime for which vapor cavities and bubbles reach the end of the channel. It is sometimes defined as the regime from which cavitation starts to improve atomization processes, even if the related 
mechanisms are not well highlighted (increase in turbulence Tamaki et al. (2001); He and Ruiz (1995) or interface deformation Sou et al. (2007) induced by bubble collapses). In Sou et al. study Sou et al. (2007), the starting point of super-cavitation regime is associated to a normalized cavity length $l_{\text {norm }} \simeq 70 \%$, that is a significantly larger value than the one suggested by Figure 16c. The starting point could be explained by the up-scaled configuration studied in Sou et al. (2007) or by a gap between the starting point of structure velocity fluctuation enhancement and that of a significant effect in atomization efficiency (or change in spray angle). In the following, channel height has been modified in order to know if it affects structure velocity fluctuations at the outlet.

\subsection{Channel height influence on outlet structure velocity fluctuations}

Changing channel height without modifying other parameters is a difficult task. Indeed, cavitation is very sensitive to small geometric modifications, especially at the channel inlet. A small change in the inlet radius or the presence of a defect at this point can dramatically influence cavitation inception. In order to keep the same inlet geometry, the different channels are constructed with the same pair of metal sheets. The space between the top sheet and the bottom sheet is modified by means of wedges in paper gasket placed between the metal sheets (Figure 1b). The use of paper gasket ensures the sealing of the metal sheets. As the paper gasket is compressible, it is not possible to precisely predict the height of the channel. The geometry of the different channels is therefore measured a posteriori by a SEM. Three different channel heights are obtained, namely 238, 322 and $388 \mu \mathrm{m}$. The streamwise and cross-streamwise relative root mean squares of structure velocity are calculated in the same way as explained in Section 4.3 for the three different channels in a flow area of $60 \mathrm{px} \times 60 \mathrm{px}, 80 \mathrm{px} \times 80 \mathrm{px}$ and 96 px $\times 96$ px, respectively.

Figure 17 presents the structure velocity fluctuations $\varsigma_{x}$ and $\varsigma_{y}$ versus $l_{\text {norm }}$ for different channel heights. The higher the channel, the more significant the velocity fluctuations. The increase in structure velocity fluctuations is significant in the cross-streamwise direction, suggesting an anisotropy of the turbulent flow. The channel height has no influence on the critical length $l_{\text {norm }}$ at which fluctuations increase suddenly: for the three channel heights and both directions, this critical length is still $l_{\text {norm }}=40$ $50 \%$. The turbulence induced by cavitation seems to be more developed in 
higher nozzles, but the critical normalized cavity length defining the supercavitation regime starting point seems to be independent of the channel height, in the size range considered here. The effect of a complete scaleup (including the channel length) has not been considered.

The potential effect of temperature on structure velocity fluctuations has been investigated for flow temperatures varying between $20^{\circ} \mathrm{C}$ and $50^{\circ} \mathrm{C}$. The results are not detailed in this paper because no significant effect has been observed within this temperature range.

\section{Conclusion}

Shadowgraph-like imaging can be used to investigate a cavitating flow in a micro-channel. This optical technique provides a wealth of information on the flow, allowing one to distinguish vapor and liquid phases, providing qualitative information on density gradients in the liquid phase. In particular, grey-level random-like structures are visible in regions when and for flow conditions where turbulence is expected. These structures are likely related to density fluctuations of the turbulent flow. Although their connection with the turbulent structures is not rigorously established, the grey-level structures have been studied by using space-time correlations and spectral analysis. In single-phase flow condition (for low pressure drop from upstream to downstream of the channel), the integral length scale deduced from the space-correlation of the grey-level structures decreases with increasing Reynolds number (or pressure drop). At cavitation inception, it stops decreasing and increases slowly. A Fourier transform analysis of these structures shows a spectrum enlargement with increasing Reynolds number. Before cavitation inception, the spectra are essentially shifted toward small scales when the largest scales increase slowly. After cavitation inception, a more significant growth rate of the largest scales is observed, suggesting a production of large scale turbulent structures induced by cavitation

- although, as indicated above, the connection between the grey-level structures and turbulence is not rigorously established yet. The most important results provided by the space-time correlation analysis of the shadowgraph-like images is that grey-level structures remain correlated between two images recorded at $300 \mathrm{~ns}$ delay. Advection velocities of the structures are then deduced from space-time correlations. These structure velocities are close to the velocities deduced from flow rate measurements. In addition, an image correlation algorithm, similar to those currently 
employed in Particle Image Velocimetry (PIV), can be applied to couples of shadowgraph-like images to obtain mean velocity fields as well as velocity fluctuations in the channel flow. The main advantage of this technique is that it does not use seeding particles which could act as cavitation nuclei and modify the flow behavior. The structure velocity fields obtained are only partial as on the one hand, the presence of grey-level structures is required to extract velocity information and, on the other hand, velocity cannot be measured in flow areas where vapor cavities are fully developed. However, mean structure velocity profiles have been measured in various flow sections. The flow rate deduced from these profiles near the channel exit are consistent with the flowmeter measurement, with a nearly constant deviation in the order of $5 \%$ from low pressure drop conditions to choked flow condition for which larger deviations are observed. Assuming that the greylevel structure velocity is representative of the real flow velocity, structure velocity fluctuations have been also evaluated as a function of the pressure drop from upstream to downstream of the channel. The relative structure velocity fluctuation increases slowly with increasing Reynolds number in single-phase conditions and also after cavitation inception, until the supercavitation regime for which a fast increase in the fluctuation growing rate is observed. The critical cavity length defining the starting point of the supercavitation regime is evaluated to be about half the channel height $\left(l_{\text {norm }} \simeq 40\right.$ $50 \%$ ). This value is smaller than the one reported in Sou et al. (2007) study $\left(l_{\text {norm }} \simeq 70 \%\right)$ for a similar but up-scaled configuration. Variations of the channel height (between $238 \mu \mathrm{m}$ and $388 \mu \mathrm{m}$ ) and flow temperature (between $20^{\circ} \mathrm{C}$ and $50^{\circ} \mathrm{C}$ ) have also been investigated. No significant temperature effects have been observed. Greater relative structure velocity fluctuations have been found in higher channels but the critical length associated to supercavitation regime does not change in the considered range of channel heights.

\section{Acknowlegments}

This work takes place in the French collaborative program NADIAbio (New Advance Diesel Injection Diagnosis for bio fuels). This program is supported by the French Automotive Cluster Mov'eo, and funded by the DGCIS (Direction Générale de la Compétitivité, de l'Industrie et des Services), the Région Haute Normandie and the Conseil Général des Yvelines.

Bergwerk, W., 1959. Flow pattern in diesel nozzle spray holes. Proceedings of 
the Institution of Mechanical Engineers 1847-1982 (vols 1-196) 173 (1959), 655-660.

Birouk, M., Lekic, N., 2009. Liquid jet breakup in quiescent atmosphere: A review. Atomization and Sprays 19 (6), 501-528.

Chaves, H., 2008. Particle image velocimetry measurements of the cavitating flow in a real size transparent VCO nozzle. In: ILASS 2008.

Chorążewski, M., Dergal, F., Sawaya, T., Mokbel, I., Grolier, J., Jose, J., 2012. Thermophysical properties of normafluid (iso 4113) over wide pressure and temperature ranges. Fuel.

Gorokhovski, M., Herrmann, M., 2008. Modeling primary atomization. Annu. Rev. Fluid Mech. 40, 343-366.

He, L., Ruiz, F., 1995. Effect of cavitation on flow and turbulence in plain orifices for high-speed atomization. Atomization and Sprays 5 (6), 569-584.

Hiroyasu, H., 1991. Break-up length of a liquid jet and internal flow in a nozzle. ICLASS-91, 275-282.

Hiroyasu, H., 2000. Spray breakup mechanism from the hole-type nozzle and its applications. Atomization and Sprays 10 (3-5), 511-527.

Iben, U., Morozov, A., Winklhofer, E., Wolf, F., 2011. Laser-pulse interferometry applied to high-pressure fluid flow in micro channels. Experiments in fluids 50 (3), 597-611.

Kim, J., Hussain, F., 1992. Propagation velocity and space-time correlation of perturbations in turbulent channel flow. NASA STI/Recon Technical Report N 93, 25082.

Lebas, R., Menard, T., Beau, P., Berlemont, A., Demoulin, F.-X., 2009. Numerical simulation of primary break-up and atomization: Dns and modelling study. International Journal of Multiphase Flow 35 (3), 247260 .

Marcer, R., Dassibat, C., Argueyrolles, B., 2008. Simulation of two-phase flows in injectors with the cfd code eole. 
Mauger, C., Méès, L., Michard, M. Azouzi, A., Valette, S., 2012. Shadowgraph, schlieren and interferometry in a $2 \mathrm{~d}$ cavitating channel flow. Experiment in Fluids 53, 1895-1913.

Ménard, T., Tanguy, S., Berlemont, A., 2007. Coupling level set/vof/ghost fluid methods: Validation and application to $3 \mathrm{~d}$ simulation of the primary break-up of a liquid jet. International Journal of Multiphase Flow 33 (5), $510-524$.

Ndiaye, E., Bazile, J., Nasri, D., Boned, C., Daridon, J., 2012. High pressure thermophysical characterization of fuel used for testing and calibrating diesel injection systems. Fuel.

Nurick, W., 1976. Orifice cavitation and its effect on spray mixing. Journal of fluids engineering 98,681 .

Soteriou, C., Andrews, R., Smith, M., 1995. Direct injection diesel sprays and the effect of cavitation and hydraulic flip on atomization. Tech. rep., Society of Automotive Engineers, 400 Commonwealth Dr, Warrendale, PA, 15096, USA,.

Sou, A., Hosokawa, S., Tomiyama, A., 2007. Effects of cavitation in a nozzle on liquid jet atomization. International journal of heat and mass transfer 50 (17), 3575-3582.

Sou, A., Maulana, M., Hosokawa, S., Tomiyama, A., 2008. Ligament formation induced by cavitation in a cylindrical nozzle. Journal of Fluid Science and Technology 3 (5), 633-644.

Stanley, C., Rosengarten, G., Milton, B., Barber, T., 2008. Investigation of cavitation in a large-scale transparent nozzle. University of New South Wales, Australia, F.

Tamaki, N., Shimizu, M., Hiroyasu, H., 2001. Enhancement of the atomization of a liquid jet by cavitation in a nozzle hole. Atomization and Sprays 11 (2), 125-137.

Tseng, Q., Duchemin-Pelletier, E., Deshiere, A., Balland, M., Guillou, H., Filhol, O., Théry, M., 2012. Spatial organization of the extracellular matrix regulates cell-cell junction positioning. Proceedings of the National Academy of Sciences 109 (5), 1506-1511. 
Winklhofer, E., Kull, E., Kelz, E., Morozov, A., 2001. Comprehensive hydraulic and flow field documentation in model throttle experiments under cavitation conditions. In: ILASS-Europe. Vol. 10. pp. 71-73.

Wu, P., Miranda, R., Faeth, G., 1995. Effects of initial flow conditions on primary breakup of nonturbulent and turbulent round liquid jets. Atomization and Sprays 5 (2). 


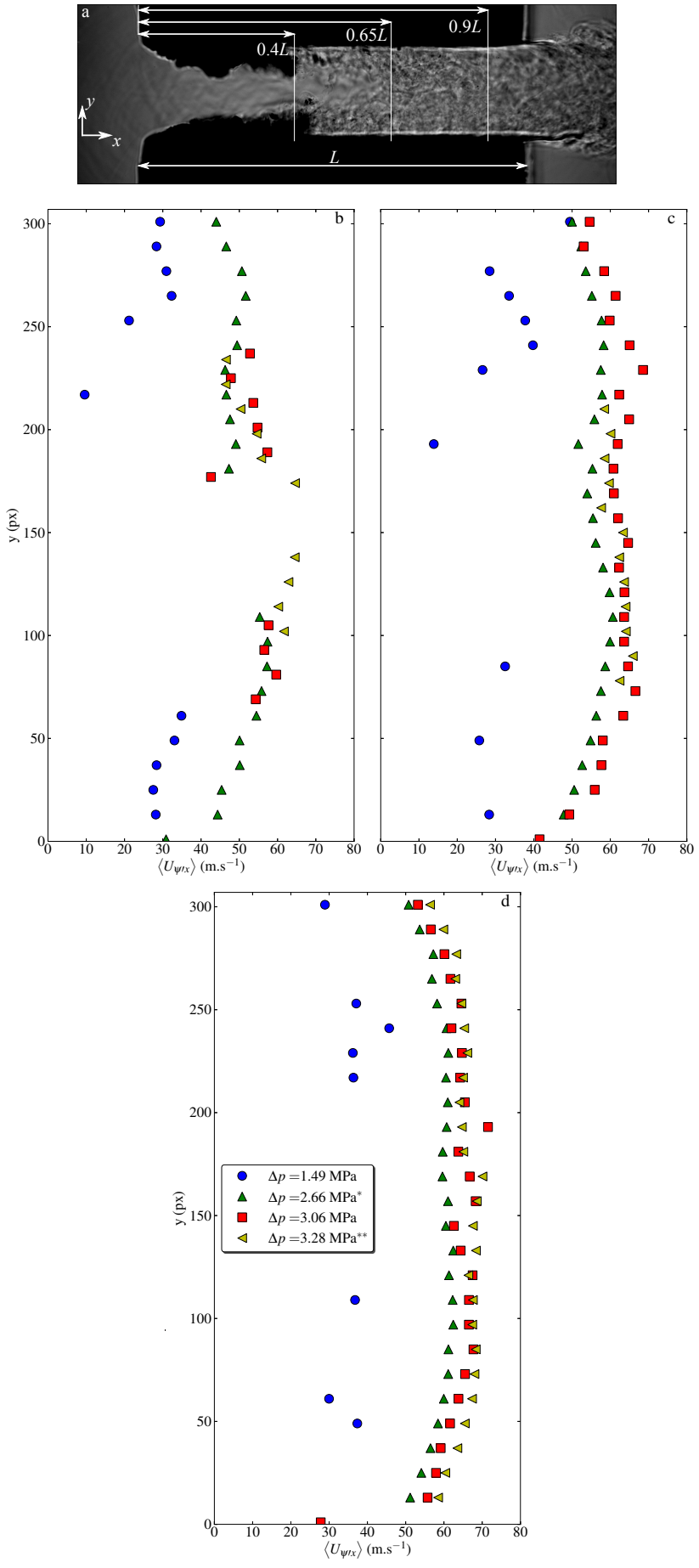

Figure 12: a: Example of flow visualization $\Delta p=3.12 \mathrm{MPa}$. Streamwise velocity profiles $\left\langle U_{\psi^{\prime} x}\right\rangle$ of grey-level structures for different flow conditions $\Delta p$ and different channel heights (b: $0.4 L$. c: $0.65 L$. d: $0.9 L$ ). The channel lierght is $388 \mu \mathrm{m}$. $p_{u p}=5.00 \mathrm{MPa}, T=24^{\circ} \mathrm{C}$. ${ }^{*}$ Cavitation inception. ${ }^{*}$ Super-cavitation. 

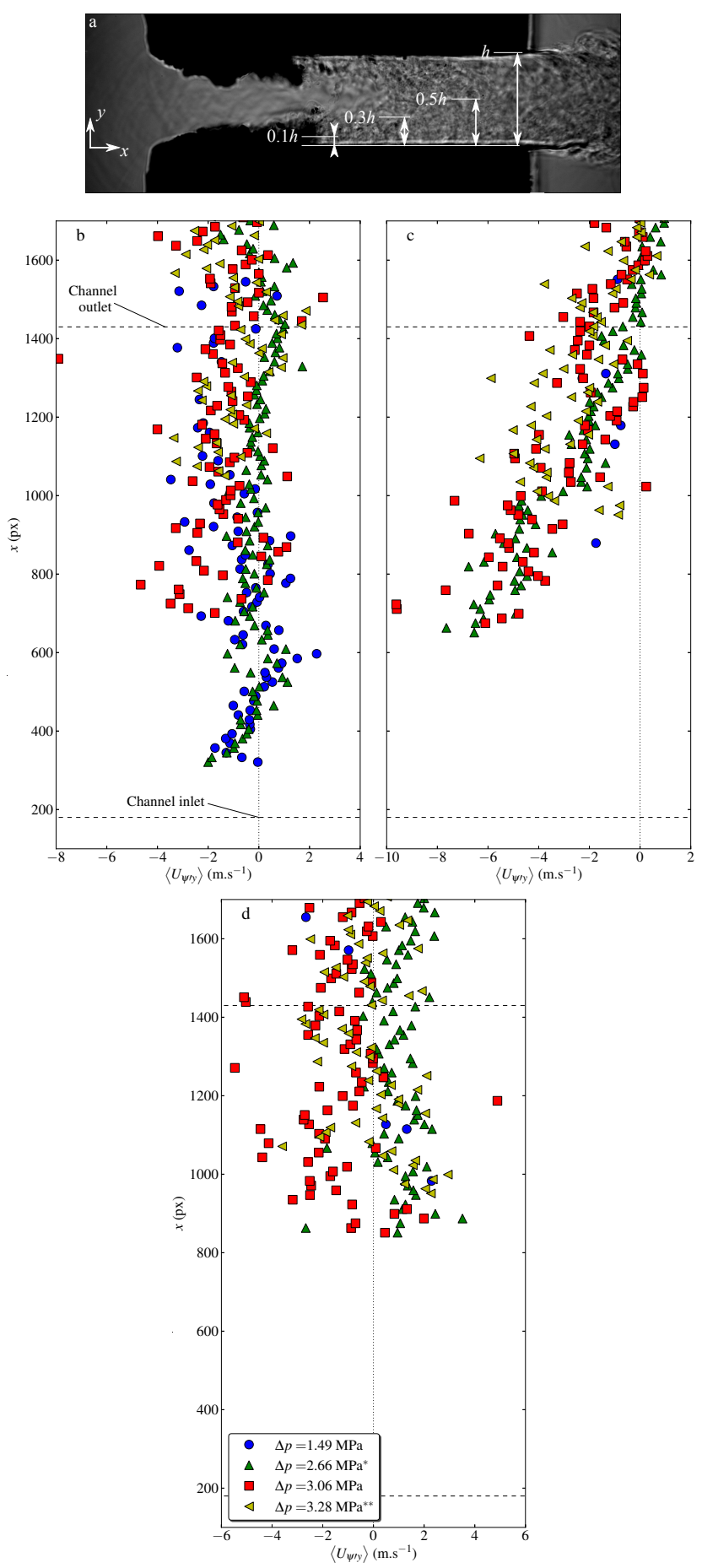

Figure 13: a: Example of flow visualization $\Delta p=3.12 \mathrm{MPa}$. Cross-streamwise velocity profiles $\left\langle U_{\psi^{\prime} y}\right\rangle$ of grey-level structures for different flow conditions $\Delta p$ and different channel heights (b: $0.1 h$. c: $0.3 h$. d: $0.5 h$ ). The 2 annel height is $388 \mu \mathrm{m}$. $p_{u p}=5.00 \mathrm{MPa}$, $T=24^{\circ} \mathrm{C} .{ }^{*}$ Cavitation inception. ${ }^{* *}$ Super-cavitation. 


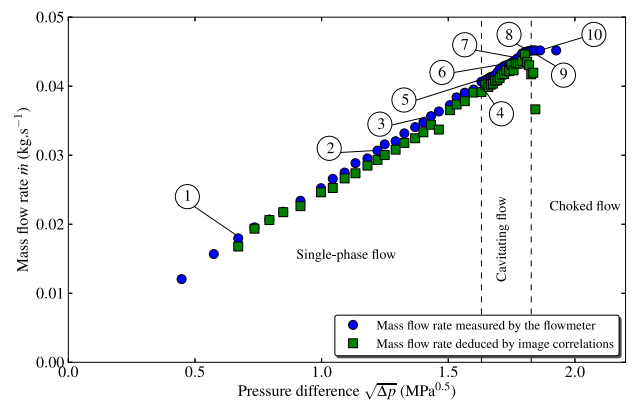

Figure 14: Comparison between the mass flow rate measured by the flowmeter and the mass flow rate deduced from the image correlations. The channel height is $388 \mu \mathrm{m}$. $p_{u p}=5.00 \mathrm{MPa}, T=24^{\circ} \mathrm{C}$.

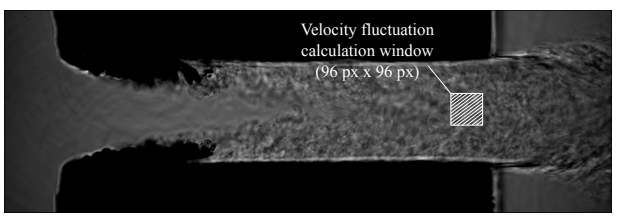

Figure 15: Calculation window for velocity fluctuations in a $388 \mu \mathrm{m}$ high channel. $p_{u p}=5.00 \mathrm{MPa}, \Delta p=2.99 \mathrm{MPa}, T=24^{\circ} \mathrm{C}$. 


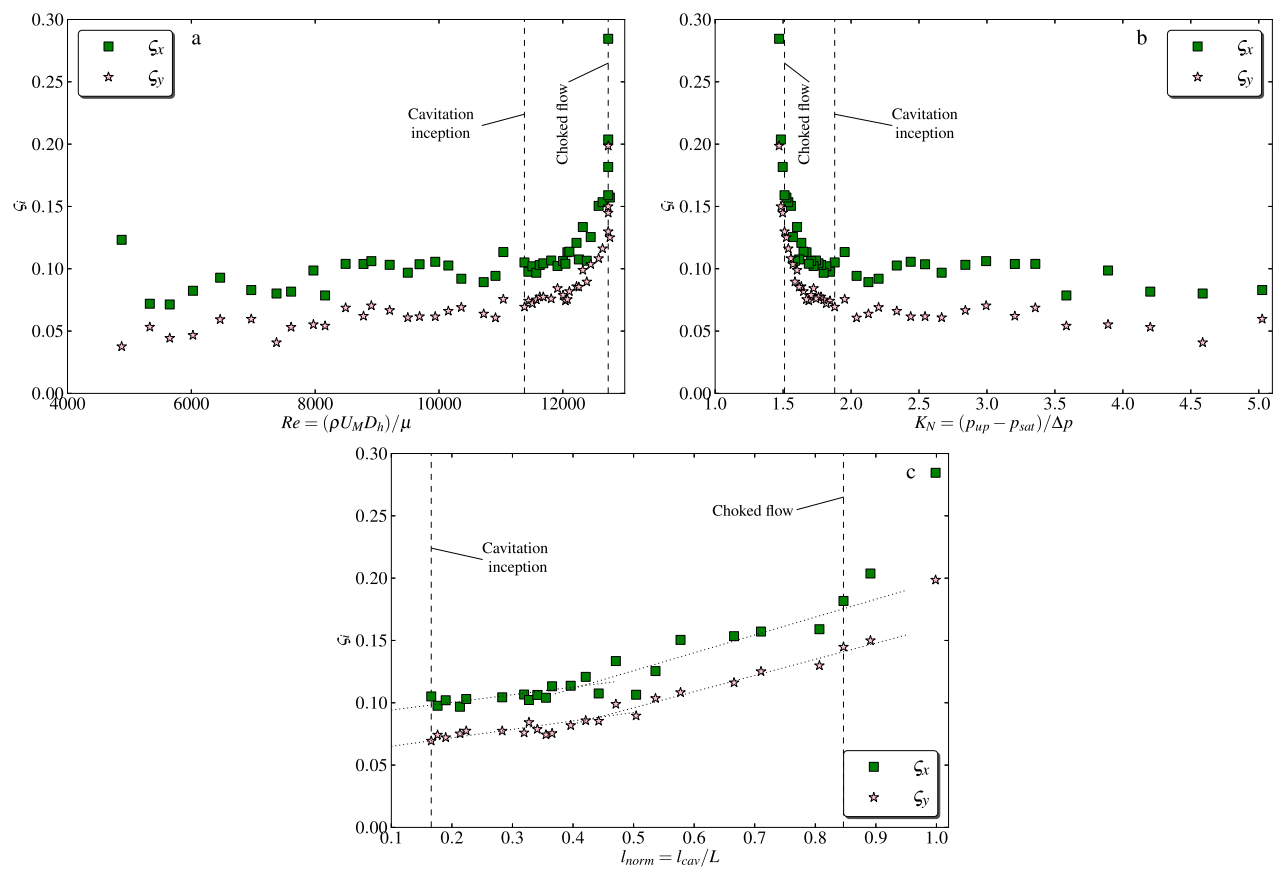

Figure 16: Relative velocity fluctuations versus Reynolds number $R e$ (a), Nurick cavitation number $K_{N}$ (b) and normalized length of vapor cavities $l_{\text {norm }}$ (c). The channel height is $388 \mu \mathrm{m} . p_{u p}=5.00 \mathrm{MPa}, T=24^{\circ} \mathrm{C}$.
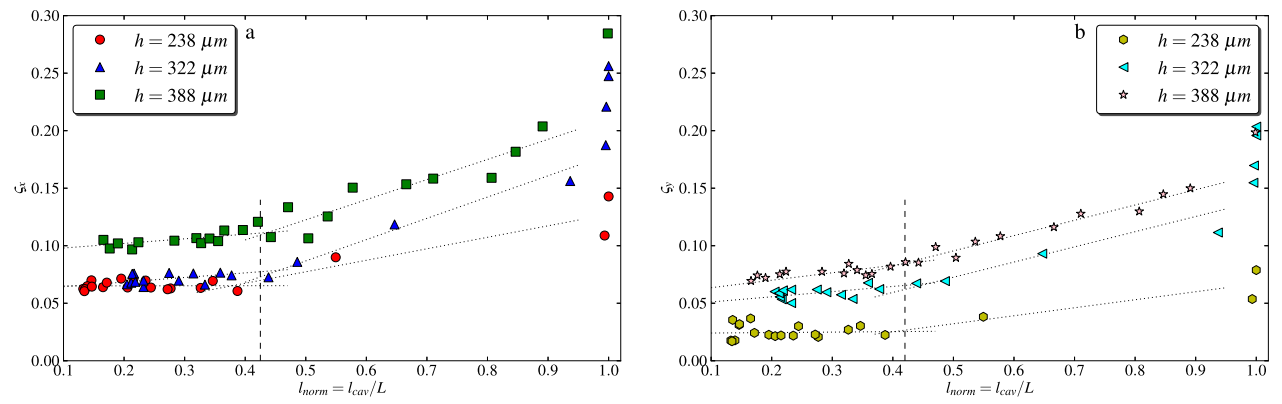

Figure 17: Relative velocity fluctuations versus normalized length of vapor cavities $l_{\text {norm }}$ for different channel heights. $p_{u p}=5.00 \mathrm{MPa}, T=24^{\circ} \mathrm{C}$. 